\title{
Exclusion Statistics of Quasiparticles in Condensed States of Composite Fermion Excitations
}

\author{
Piotr Sitko \\ Institute of Physics, Wroctaw University of Technology, \\ Wybrzeże Wyspiańskiego 27, 50-370 Wroctaw, Poland.
}

\begin{abstract}
The exclusion statistics of quasiparticles is found at any level of the hierarchy of condensed states of composite fermion excitations (for which experimental indications have recently been found). The hierarchy of condensed states of excitations in boson Jain states is introduced and the statistics of quasiparticles is found. The quantum Hall states of charged $\alpha$-anyons ( $\alpha$ - the exclusion statistics parameter) can be described as incompressible states of $(\alpha+2 p)$-anyons $(2 p-$ an even number $)$.
\end{abstract}

Recent fractional quantum Hall experiments by Pan et al. [1] (described in [2]) indicate the presence of the new odd-denominator hierachical states (other than Laughlin and Jain states), also indications of even-denominator hierarchical states $[1,3]$ are found. If the results are confirmed they will bring a breaktrough in our knowledge of the fractional quantum Hall effect. The hierarchy of odd-denominator states in the fractional quantum Hall effect was introduced by Haldane at early stages of the quantum Hall theory [4] (short time after Laughlin presented his original approach [5]). Within years, however, the experiments supported only hierarchical states given by the composite fermion approach. The composite fermion approach predicts the so-called Jain states at filling fractions of the form $\nu=\frac{n_{0}}{2 p_{0} n_{0}+\beta_{0}}$ (where $2 p_{0}$ - the Chern-Simons composite fermion parameter being an even number, $n_{0}$ - the number of effective shells filled, $\beta_{0}$ - the sign of the effective field with respect to the external magnetic field). The hierachical theory of condensed states of composite fermion excitations was also proposed (in a close analogy with the Haldane hierarchy), but hierachical states were not numerically verified [6] (e. g. the 4/11 state - for which the strongest evidence is found [1]). The experiment [1] opens the possibility for a realization of condensed states of composite fermion excitations [7].

The anyon statistics parameters of quantum Hall quasiparticles were determined by Halperin within the Haldane hierarchy picture [8]. Here, we present the analogous approach, however, the quasiparticle statistics is determined within the generalized exclusion statistics approach for condensed states of excitations in Jain states on a sphere. In Laughlin and Jain fermion states quasiparticles obey fractional statistics $[8,9]$ with exclusion statistics parameters defined earlier [10-14]. Let us add that recently it was proposed that quantum Hall quasiparticles obeying fractional statistics may serve as a good tool for quantum computation [15] (called topological quantum computation [16]).

In this paper we will determine the statistics of quasiparticles in hierarchical states using the composite fermion approach (and folowing the construction of the hierarchy of condensed states of composite fermion excitations [7]). We make use of the generalized Haldane exclusion statistics $[17,18]$ (with the statistics parameter $\alpha$ ). The anyon statistics parameter of quantum Hall quasiparticles is rederived as $\frac{\theta}{\pi}=\alpha(\bmod 2)-$ see $[18,19]$ ( $\theta$ is the phase change which appears in the wave function when two anyons are interchanged).

The generalized exclusion statistics for identical particles can be expressed in terms of the number of manyparticle states as [18]:

$$
\left(\begin{array}{c}
k+\left(\begin{array}{c}
1-\alpha \\
N
\end{array}\right)(N-1) \\
N
\end{array}\right)
$$

where $k$ is the number of available single-particle states and $\alpha$ is the statistics parameter, $N$ - the number of particles. So that, we are going to define the number of many-particle states for quasiparticles in hierarchical states using the composite fermion approach for a spherical system [20] (for excitations in Laughlin and Jain states it was done in [10-14]). The quasiparticles in even-denominator quantum Hall states are predicted to obey nonabelian fractional statistics $[6,21]$ and are not considered here.

The composite fermion approach was extensively and successfully tested in many numerical studies of quantum Hall systems - i. e. of systems of electrons in a magnetic field such that the lowest Landau level is partially filled (see for example [6]). The numerical results are available only for a small number of electrons (usually up to 12-14 electrons) and there are only data for a few quasiparticles [7]. In fact, the 4/11 state is not predicted in numerical studies for Coulomb interaction [6] (it is important to 
add that the experiment [1] indicates that the observed $4 / 11$ state is fully spin polarized).

The composite fermion hierarchy consists in the assumption that quasiparticles (e. g. in Laughlin states) can be treated in the same way as electrons (partially filling the lowest Landau level) are treated. So that, we can use the composite fermion approach (well established for electrons partially filling the lowest Landau level [6]) to quasiparticles partially filling a shell (all predictions will be the same as for electrons).

Let us introduce the hierarchy of condensed states of composite fermion excitations (following [7]). The hierarchy fractions is defined by the set of equations:

$$
\nu_{0}^{-1}=2 p_{0}+\frac{\beta_{0}}{n_{0}+\nu_{1}}
$$

where $\nu_{1}$ is the fraction in which the next $\left(n_{0}+1\right)$-th effective shell is filled. The procedure (2) can be repeated on $\nu_{1}$, and so on, to find the final odd-denominator filling fraction:

$$
\nu_{0}^{-1}=2 p_{0}+\frac{\beta_{0}}{n_{0}+\ldots+\frac{1}{2 p_{k}+\frac{\beta_{k}}{n_{k}}}} .
$$

The hierarchy of even denominator fractions is found when $\beta_{k}=0$ at any step of the hierarchy [3]. The quantum Hall states at those fractions would be related to Pfaffian states of quasiparticles (see for example $[21,6]$ ). Still, in order to define the Pfaffian states one needs the statistics of quasiparticles.

In the case of a spherical system the composite fermion effective field is given by [6]:

$$
2 S^{*}=\beta_{0}\left(2 S-2 p_{0}\left(N_{e}-1\right)\right)
$$

where $2 S$ is the number of flux quanta piercing the sphere, $N_{e}$ - the number of electrons, $2 S^{*}$ - the effective composite fermion field, $\beta_{0}$ is the sign of the effective field with respect to $2 S$. One has also the relations: $2 S_{q e(0)}=2 S^{*}+2 n_{0}, 2 S^{*} n_{0}+n_{0}^{2}=N_{e}-N_{q e(0)}$ (if only quasielectrons are present).

The number of many-particle states (at any step of the hierarchy) is given by:

$$
\left(\begin{array}{c}
2 S_{q e}+1 \\
N_{q e}
\end{array}\right) .
$$

One can also perform the composite fermion transformation for this effective shell

$$
2 S_{q e}^{*}=\beta_{q e}\left(2 S_{q e}-2 p_{q e}\left(N_{q e}-1\right)\right),
$$

$\beta_{q e}$ is the sign of the effective quasiparticle shell (with respect to $2 S_{q e}$ ).
Let us first find the statistics parameters of quasiparticles in Jain states $[12,13]$, and we will determine the statistics of quasiparticles in the next step of the hierarchy. Using the definition (1) one can write

$$
2 S_{q e(0)}=\left(1-\alpha_{q e(0)}\right) N_{q e(0)}+A\left(2 S, n_{0}, 2 p_{0}, \beta_{0}\right)
$$

where $A$ is a function of $2 S, n_{0}, 2 p_{0}, \beta_{0}[12,13]$ :

$$
A=\beta_{0} 2 S+\beta_{0} 2 p_{0}+2 n_{0}-\beta_{0} 2 p_{0} \frac{\beta_{0} n_{0}\left(2 S+2 p_{0}\right)+n_{0}^{2}}{1+2 p_{0} \beta_{0} n_{0}},
$$

and $1-\alpha_{q e(0)}=\frac{-\beta_{0} 2 p_{0}}{1+2 p_{0} \beta_{0} n_{0}} \quad$ (as was found in $\left.[12,13]\right)$. One gets next

$$
2 S_{q e(0)}^{*}=\beta_{1}\left(2 S_{q e(0)}-2 p_{1}\left(N_{q e(0)}-1\right)\right),
$$

and $2 S_{q e(1)}=2 S_{q e(0)}^{*}+2 n_{1}$. The number of many particle states for $N_{q e(1)}$ is given by $\left(\begin{array}{c}2 S_{q e(1)}+1 \\ N_{q e(1)}\end{array}\right)$. We need also the relation (if only quasielectrons are present): $N_{q e(0)}-N_{q e(1)}=2 S_{q e(0)}^{*} n_{1}+n_{1}^{2}$, (or if only quasiholes are present) $N_{q e(0)}+N_{q h(1)}=2 S_{q e(0)}^{*} n_{1}+n_{1}^{2}$. Note that here we perform the composite fermion transformation only on quasielectrons (not on quasiholes - we use the $\beta=-1$ effective field instead [3]). The final result is:

$$
\frac{1}{1-\alpha_{q e(1)}}=\frac{1}{\beta_{1}\left(1-\alpha_{q e(0)}-2 p_{1}\right)}-n_{1}=\frac{1}{\alpha_{q h(1)}-1}
$$

We can also write the general relation (for quasiparticles at the $k$-step of the hierachy):

$$
\frac{1}{1-\alpha_{q e(k)}}=\frac{1}{\beta_{k}\left(1-\alpha_{q e(k-1)}-2 p_{k}\right)}-n_{k},
$$

and $\alpha_{q h(k)}-1=1-\alpha_{q e(k)}$. One can notice that the statistics of composite fermion excitations in Jain states can be found by putting $\alpha_{q e(-1)}=1$ (simply because electrons are fermions). It can be rewritten as $\alpha_{q e(0)}=$ $1+\frac{\beta_{0} 2 p_{0}}{1+\beta_{0} n_{0} 2 p_{0}}$ and for quasiholes in Jain states $\alpha_{q h(0)}=$ $1-\frac{\beta_{0} 2 p_{0}}{1+\beta_{0} n_{0} 2 p_{0}}$ in agreement with previous results $[12,13]$.

We can define the statistics parameter at any level of the hierarchy by the set of equations

$$
\begin{gathered}
\frac{1}{1-\alpha_{q e(0)}}=\frac{1}{-\beta_{0} 2 p_{0}}-n_{0} \\
\frac{1}{1-\alpha_{q e(1)}}=\frac{1}{\beta_{1}\left(1-\alpha_{q e(0)}-2 p_{1}\right)}-n_{1} \\
\frac{1}{1-\alpha_{q e(2)}}=\frac{1}{\beta_{2}\left(1-\alpha_{q e(1)}-2 p_{2}\right)}-n_{2}
\end{gathered}
$$




$$
\frac{1}{1-\alpha_{q e(k)}}=\frac{1}{\beta_{k}\left(1-\alpha_{q e(k-1)}-2 p_{k}\right)}-n_{k}
$$

and $1-\alpha_{q e(k)}=\alpha_{q h(k)}-1$.

The Haldane hierachy corresponds to the choice $n_{k}=$ 1 at every step of the hierarchy (3) [7], then one can find the result (which is analogous to the Eq. (4) of Ref. [8]):

$$
\frac{\beta_{k}}{\alpha_{q h(k)}}=\left(2 p_{k}+1+\beta_{k}\right)-\alpha_{q h(k-1)} .
$$

Note again that we use the $\beta=-1$ effective field instead of considering condensed states of quasiholes. One can easily notice that the denominator in $\alpha_{q h(k-1)}$ is the numerator in $\alpha_{q h(k)}$ (for the Haldane hierarchy). The Jain sequence within the Haldane hierarchy is found for $p_{k}=0$ and $n_{k}=1, \beta_{k}=1$, at any step.

Let us consider the second order of the hierarchy. For the $4 / 11$ state $\left(p_{0}=1, n_{0}=1, \beta_{0}=1, p_{1}=1, n_{1}=1\right.$, $\left.\beta_{1}=1\right)$ one finds $\alpha_{q h(1)}(\nu=4 / 11)=\frac{3}{11}$ and $\alpha_{q e(1)}(\nu=$ $4 / 11)=2-\frac{3}{11}$. For several other fractions the statistics parameters are given in Table I. One can observe that (in agreement with results of Halperin [8]) $\alpha_{q h(k)}=p / q$ where $p$ is the denominator of the parent state and $q$ is the denominator of the daughter state. For $\nu_{0}=5 / 13$ the parent state (in the spirit of the Haldane hierarchy) is the $2 / 5$ state (to get it one can put $n_{1}=1$ in the second row of Table I), for $\nu_{0}=5 / 17$ the parent state is the $2 / 7$ state $\left(n_{1}=1\right)$. The $31 / 65$ state is the daughter state of the Jain $10 / 21$ state [1].

Since we perform the composite fermion transformation only on quasielectrons the $4 / 13$ state is found with $p_{0}=2$ and $\beta_{0}=-1\left(n_{0}=1\right)$ for the Laughlin $1 / 3$ state. One can also put $p_{0}=1, \beta_{0}=1, n_{0}=1$, to get the Laughlin $1 / 3$ state within the composite fermion approach. The excitations above the $1 / 3$ state can also be described in two ways (both descriptions can be to some extent verified numerically - see for example [22]). For example the $2 / 7$ state can be seen as the $1 / 3$ state of quasiholes (within the $p_{0}=1$ description) or, with $p_{0}=2$, it is the Jain state (first order of the hierachy, or $\nu_{1}=1$ - quasielectrons completely fill the shell $2 S_{q e}$ ). It is apparent that numbers of many-particle states for quasiparticles in the two descriptions differ [22]. The lowest energy states within the $p_{0}=1$ description can be seen as states of quasielectrons within the $p_{0}=2$ description [22]. Specifically at $\nu_{0}=2 / 7$ one gets the single many-particle state of $\left(p_{0}=2\right)$ quasielectrons (the ground state) and $\left(\begin{array}{c}3\left(N_{q h(0)}-1\right)+1 \\ N_{q h(0)}\end{array}\right)$ many-particle states of $\left(p_{0}=1\right)$ quasiholes. Of course, when one would perform the composite fermion transformation on those quasiholes one would find the single-many particle state (the ground state at $\left.\nu_{0}=2 / 7\right)$. We argue then that different descriptions of excited states are related through the composite fermion transformation. Quasiparticles within different descriptions are related by (we consider quasiholes $\left(p_{k}=p\right)$ and corresponding quasielectrons $\left.\left(p_{k}=p+1\right)\right)$ :

$$
\alpha_{q e(k)}^{\left(p_{k}=p+1, \beta_{k}=-1, n_{k}=1\right)}-\alpha_{q h(k)}^{\left(p_{k}=p, \beta_{k}=1, n_{k}=1\right)}=2
$$

which exactly corresponds to the composite fermion transformation on quasiparticles. For example Laughlin quasiholes $\left(p_{0}=1, \alpha_{q h(0)}=1 / 3\right)$ correspond to quasielectrons with $p_{0}=2$ and $\alpha_{q e(0)}=7 / 3$. The anyon statistics parameter within different descriptions equals $\theta / \pi=\alpha(\bmod 2)$. In general the composite fermion transformation on quasiparticles ( $\alpha$-anyons) would give "superanyons" of statistics $\alpha+2 p$ ( $2 p$ is an even number). This corresponds to the description of the system of electrons in terms of composite fermions $[18,19,13]$ (called "superelectrons" by $\mathrm{Wu}[18]$ ).

There is a growing interest in studies of quantum Hall boson systems (expected to be found in rotating Bose gases [24]). Below we define the hierarchical states in boson quantum Hall systems for which $\nu_{F}^{-1}=\nu_{B}^{-1}+1\left(\nu_{F}\right.$ is the filling fraction for fermions, $\nu_{B}$ - the corresponding filling fraction for bosons [25]). We do not consider here the Pfaffian boson states [24] (or Read and Reazyi states $[21,24])$. Within the above approach we only have to change $2 p_{0}$ into $2 p_{0}-1$ and other relations in (11) remain the same. For the Jain boson states one finds:

$$
\frac{1}{1-\alpha_{q e(0)}}=\frac{1}{\beta_{0}\left(1-2 p_{0}\right)}-n_{0}=\frac{1}{\alpha_{q h(0)}-1} .
$$

For example at $\nu_{B}=\frac{1}{2}, \alpha_{q e(0)}\left(\nu_{B}=\frac{1}{2}\right)=\frac{3}{2}, \alpha_{q h(0)}\left(\nu_{B}=\right.$ $\left.\frac{1}{2}\right)=\frac{1}{2}$. For the hierarchical state (the condensed state of excitations in the Laughlin boson state $\left.\nu_{B}=1 / 2\right) \nu_{B}=$ $4 / 7$ (corresponding to the $\nu_{F}=4 / 11$ fermion state) one gets $\alpha_{q h(1)}\left(\nu_{B}=4 / 7\right)=\frac{2}{7}$. For several other hierachical boson fractions the statistics of quasiparticles is given in Table II. The parent state for the boson fillings $4 / 7$, $6 / 11,4 / 9$ (in the spirit of the Haldane hierarchy) is the Laughlin $1 / 2$ boson state. The parent states for $5 / 8$ and $5 / 12$ are $2 / 3$ and $2 / 5$, respectively. For $\nu_{B}=31 / 34$ the parent state is $\nu_{B}=10 / 11$.

We want to underline that the relation (10) satisfies the symmetry $Q E\left(2 p_{0}, \beta_{0}, n_{0}, \quad \ldots, 2 p_{k}, \beta_{k}, n_{k}\right)-$ $Q H\left(2 p_{0}, \beta_{0}, n_{0}, \ldots, 2 p_{k}, \beta_{k}, n_{k}+1\right)$ (all parameters in parentheses are the same except for $n_{k}$, for bosons $\left.2 p_{0} \longrightarrow\left(2 p_{0}-1\right)\right)$ : 


$$
\alpha_{q e}\left(n_{k}\right)=\alpha_{q h}^{-1}\left(n_{k}+1\right) .
$$

which is the result of the particle-hole conjugation (quasielectrons and quasiholes are in the same effective shell) - this corresponds to the duality described by Nayak and Wilczek $[23,13]$. The Eq. (14) is identical with the Eq. (12) for $p_{k}=0$ and $\beta_{k}=1$. Also the equation (14) is valid for the special case when $n_{k}=0$. For $k=0$ one finds $\alpha_{q e}\left(n_{0}=0\right)=2 p_{0}+1\left(\beta_{0}=1\right)-$ the exclusion statistics of composite fermions $[18,19]$. For $k>0$ one finds "superanyons" - for example quasielectrons conjugated to quasiholes with the exclusion statistics parameters given in Tables (the last column). At filling fractions given in the first column of the Tables these "superanyons" fill a single effective shell (they form incompressible states).

In conclusion, we found the exclusion statistics parameter $\alpha$ for quasiparticles in odd-denominator hierarchical condensed states of composite fermion excitations on a sphere. The statistics parameters for quasiparticles in the hierarchy of condensed states of excitations in Jain boson states are also found. The statistics parameters $\alpha$ obey quasiparticle - quasihole symmetry (when they are in the same effective shell). The anyon statisics parameter for quasiparticles is obtained as $\frac{\theta}{\pi}=\alpha(\bmod 2)$. The quantum Hall states of charged anyons of the exclusion statistics parameter $\alpha$ can be described as incompressible states of $(\alpha+2 p)$-anyons.

This work was supported by KBN grant.

[1] W. Pan, H. L. Störmer, D. C. Tsui, L. N. Pfeiffer, K. W. Baldwin, K. W. West, Phys. Rev. Lett. 90 (2003) 016801

[2] J. H. Smet, Nature 422 (2003) 391

[3] P. Sitko, J. J. Quinn, D. C. Marinescu, Phys. Rev. B 56 (1997) 3606

[4] F. D. M. Haldane, Phys. Rev. Lett. 51 (1983) 606

[5] R. B. Laughlin, Phys. Rev. Lett. 50 (1983) 1395

[6] L. Jacak, P. Sitko, K. Wieczorek, A. Wójs, Quantum Hall Systems: Braid groups, composite fermions, and fractional charge, Oxford University Press, Oxford 2003, in press

[7] P. Sitko, K. S. Yi, J. J. Quinn, Phys. Rev. B 56 (1997) 12417

[8] B. I. Halperin, Phys. Rev. Lett. 52 (1984) 1583

[9] D. Arovas, J. R. Schrieffer, F. Wilczek, Phys. Rev. Lett. 53 (1984) 722

[10] S. He, X.-C. Xie, F.-C. Zhang, Phys. Rev. Lett. 68 (1992) 3460
[11] M. D. Johnson, G. S. Canright, Phys. Rev. B 49 (1994) 2947

[12] S. B. Isakov, G. S. Canright, M. D. Johnson, Phys. Rev. B 55 (1997) 6727

[13] P. Sitko, Physica E 12 (2002) 116

[14] W.-P. Su, Y.-S. Wu, J. Yang, Phys. Rev. Lett. 77 (1996) 3423

[15] D. V. Averin and V. J. Goldman, Solid State Communications, 121 (2002) 25

[16] P. Zanardi, S. Lloyd, Phys. Rev. Lett. 90 (2003) 067902

[17] F. D. M. Haldane, Phys. Rev. Lett. 67 (1991) 937

[18] Y.-S. Wu, Phys. Rev. Lett. 73 (1994) 922

[19] A. Dasniéres de Veigy, S. Ouvry, Phys. Rev. Lett. 72 (1994) 600

[20] X. M. Chen, J. J. Quinn, Solid State Commun. 92 (1994) 865

[21] N. Read, E. H. Rezayi, Phys. Rev. B 59 (1999) 8084

[22] K. S. Yi, P. Sitko, A. Khurana, J. J. Quinn Phys. Rev. B 54 (1996) 16432

[23] C. Nayak, F. Wilczek, Phys. Rev. Lett. 73 (1994) 2740

[24] N. R. Cooper, N. K. Wilkin, J. M. F. Gunn, Phys. Rev. Lett. 87 (2001) 120405

[25] X. C. Xie, S. He, S. Das Sarma, Phys. Rev. Lett. 66 (1991) 389

\begin{tabular}{|c|r|r|r|r|r|r|r|r|}
\hline \hline$\nu_{0}^{F}$ & $p_{0}$ & $n_{0}$ & $\beta_{0}$ & $\alpha_{q e(0)}$ & $p_{1}$ & $n_{1}$ & $\beta_{1}$ & $\alpha_{q h(1)}$ \\
\hline $4 / 11$ & 1 & 1 & 1 & $5 / 3$ & 1 & 1 & 1 & $3 / 11$ \\
\hline $5 / 13$ & 1 & 1 & 1 & $5 / 3$ & 1 & 2 & -1 & $5 / 13$ \\
\hline $6 / 17$ & 1 & 1 & 1 & $5 / 3$ & 2 & 1 & 1 & $3 / 17$ \\
\hline $4 / 13$ & 2 & 1 & -1 & $7 / 3$ & 1 & 1 & 1 & $3 / 13$ \\
\hline $5 / 17$ & 2 & 1 & -1 & $7 / 3$ & 1 & 2 & -1 & $7 / 17$ \\
\hline $31 / 65$ & 1 & 10 & 1 & $23 / 21$ & 1 & 1 & 1 & $21 / 65$ \\
\hline \hline
\end{tabular}

TABLE I. The exclusion statistics parameters for several filling fractions for fermion quantum Hall systems.

\begin{tabular}{|c|c|c|c|c|c|c|c|c|}
\hline \hline$\nu_{0}^{B}$ & $2 p_{0}-1$ & $n_{0}$ & $\beta_{0}$ & $\alpha_{q e(0)}$ & $p_{1}$ & $n_{1}$ & $\beta_{1}$ & $\alpha_{q h(1)}$ \\
\hline $4 / 7$ & 1 & 1 & 1 & $3 / 2$ & 1 & 1 & 1 & $2 / 7$ \\
\hline $5 / 8$ & 1 & 1 & 1 & $3 / 2$ & 1 & 2 & -1 & $3 / 8$ \\
\hline $6 / 11$ & 1 & 1 & 1 & $3 / 2$ & 2 & 1 & 1 & $2 / 11$ \\
\hline $4 / 9$ & 3 & 1 & -1 & $5 / 2$ & 1 & 1 & 1 & $2 / 9$ \\
\hline $5 / 12$ & 3 & 1 & -1 & $5 / 2$ & 1 & 2 & -1 & $5 / 12$ \\
\hline $31 / 34$ & 1 & 10 & 1 & $12 / 11$ & 1 & 1 & 1 & $11 / 34$ \\
\hline \hline
\end{tabular}

TABLE II. The exclusion statistics parameters for several filling fractions for boson quantum Hall systems. 